\title{
NONCOMMUTATIVE SYMMETRIC FUNCTIONS VII: FREE QUASI-SYMMETRIC FUNCTIONS REVISITED
}

\author{
GÉRARD H. E. DUCHAMP, FLORENT HIVERT, JEAN-CHRISTOPHE \\ NOVELLI, JEAN-YVES THIBON
}

\begin{abstract}
We prove a Cauchy identity for free quasi-symmetric functions and apply it to the study of various bases. A free Weyl formula and a generalization of the splitting formula are also discussed.
\end{abstract}

\section{INTRODUCTION}

This article is essentially an appendix to [4]. We gather here some useful properties of the algebra FQSym of free quasi-symmetric functions which were overlooked in [4].

Recall that FQSym is a subalgebra of the algebra of noncommutative polynomials in infinitely many variables $a_{i}$ which is mapped onto Gessel's algebra of quasi-symmetric functions QSym by the commutative image $a_{i} \mapsto x_{i}$ of $\mathbb{K}\langle A\rangle$. As an abstract algebra, it is isomorphic to the convolution algebra of permutations introduced by Reutenauer and his school [21, 16, 20, and further studied in [4, 14, 15, 1]. However, the realization in terms of the variables $a_{i}$ provides one with a better understanding of several aspects of the theory. For example, it becomes possible, and sometimes straigthforward, to imitate various constructions of the theory of symmetric (or quasi-symmetric) functions. An illustration is provided by the construction of the coproduct given in [4]: a free quasi-symmetric function $F$ can be regarded as a "function" of a linearly ordered alphabet $A$, and the obvious analog of the coproduct of $Q S y m$, that is, $F(A) \mapsto F\left(A^{\prime} \oplus A^{\prime \prime}\right)$, where $A^{\prime}$ and $A^{\prime \prime}$ are two mutually commuting copies of $A$, and $\oplus$ is the ordered sum, gives back the coproduct of [16].

In the present text, we further investigate the rôle of the auxiliary variables $a_{i}$.

We start with an alternative definition of the standard basis of FQSym, as resulting from a noncommutative lift of a Weyl-type character formula.

Next, we formulate a free Cauchy identity in FQSym, and investigate its implications. In the classical theory of symmetric functions, 
it is mostly in the so-called Cauchy identities, involving the product $X Y$ of two alphabets $X, Y$, that the existence of the underlying variables manifests itself. Even so, it is only when the auxiliary alphabet is specialized that one really sees the variables. Otherwise, the transformation $f(X) \mapsto f(X Y)$ is best interpreted as a coproduct, dual to the internal product. The present version involves two alphabets $A^{\prime}$, $A^{\prime \prime}$, and specializes to the noncommutative Cauchy formula of [7] un$\operatorname{der} A^{\prime} \mapsto X, A^{\prime \prime} \mapsto A$. This allows us to compute the commutative specializations of various bases of FQSym, in particular the basis $\mathbf{V}_{\sigma}$ of [4] and the basis $\mathbf{M}_{\sigma}$ of [1], recovering here the result of [1] in a simpler way.

We first illustrate the free Cauchy identity by computing the Hopf duals of various subalgebras of FQSym is a unified and straightforward way. We then apply it to the study of several multiplicative bases of FQSym and of their dual bases, including the free monomial functions of Aguiar and Sottile [1, and the basis $\mathbf{V}_{\sigma}$ introduced in [4]. We conclude with an extension of the splitting formula to FQSym.

Acknowledgements. This project has been partially supported by the grant ANR06-BLAN-0380. The authors would also like to thank the contributors of the $\mathrm{Mu}$ PAD project, and especially of the combinat part, for providing the development environment for their research (see [1] for an introduction to MuPAD-Combinat).

\section{BACKGROUND AND NOTATIONS}

Our notations are as in [4]. All algebras are over some field $\mathbb{K}$ of characteristic 0 . We shall also need the following notations.

2.1. Recall that for two permutations $\sigma$ and $\tau, \sigma \bullet \tau$ denotes their shifted concatenation. In FQSym, we set

$$
\mathbf{G}_{\sigma} \bullet \mathbf{G}_{\tau}=\mathbf{G}_{\sigma \bullet \tau}
$$

so that $I=\left(i_{1}, \ldots, i_{r}\right)$ and if $\alpha_{k} \in \mathfrak{S}_{i_{k}}$ for all $k \in[1, r]$, then

$$
\mathbf{G}_{\alpha_{1}} \ldots \mathbf{G}_{\alpha_{r}}=\left(\mathbf{G}_{\alpha_{1}} \bullet \cdots \bullet \mathbf{G}_{\alpha_{r}}\right) * S^{I} .
$$

Indeed, remembering that the internal product in the $\mathbf{G}$-basis is opposite to the product in the symmetric group $\left(\mathbf{G}_{\sigma} * \mathbf{G}_{\tau}=\mathbf{G}_{\tau \sigma}\right)$, we recognize the convolution product of permutations.

2.2. It has been shown in [8] that quasi-symmetric polynomials could be defined as the invariants of a certain action of the symmetric group, or of the Hecke algebra, called the quasi-symmetrizing action. This action will be denoted by underlining the elements, e.g., $\underline{\sigma} \cdot f$ denotes the quasi-symmetrizing action of a permutation $\sigma$ on a polynomial $f$. 


\section{The FReE Weyl Formula}

As already mentioned, $Q \operatorname{Sym}(X)$ is the algebra of $\mathfrak{S}[X]$-invariants in $\mathbb{K}[X]$ for a special action of the symmetric group, called the quasisymmetrizing action. Moreover, the analogy between Gessel's functions $F_{I}$ and Schur functions has been traced back to the existence of an expression of $F_{I}$ by a kind of Weyl character formula involving this action. In this Section, we show that this Weyl formula can be lifted to FQSym.

For a word $w$ on $\left\{a_{1}, \ldots, a_{n}\right\}$, let $|w|_{i}$ be the number of occurrences of the letter $a_{i}$ in $w$ and let the monomial $m=x^{|w|}=x_{1}^{|w|_{1}} x_{2}^{|w|_{2}} \ldots x_{n}^{|w|_{n}}$ be the commutative image of $w$. We denote by $\mathrm{C}(m)$ the composition obtained by removing the zeros in the vector $|w|$. The construction relies on the following well-known lemma (see [12, 17]):

Lemma 3.1. The map $\phi: w \mapsto\left(x^{|w|}, \operatorname{std}(w)^{-1}\right)$ is a bijection between words and pairs $(m, \sigma)$ such that $\mathrm{C}(m)$ is finer than $C(\sigma)$.

Hence, for each $\sigma$, there is a linear isomorphism $\phi_{\sigma}$ between the subspace of $\mathbb{K}\langle\langle A\rangle\rangle$ spanned by the words of standardized $\sigma$ and the subspace of $\mathbb{K}[X]$ spanned by the monomials such that $I(m) \leq C(\sigma)$.

The definitions of $F_{I}$ and $\mathbf{F}_{\sigma}$ can then be rewritten as

$$
F_{I}=\sum_{I(m) \leq I} m, \quad \text { and } \quad \mathbf{F}_{\sigma}=\sum_{I(m) \leq C(\sigma)} \phi^{-1}(m, \sigma)=\phi_{\sigma}^{-1}\left(F_{I}\right)
$$

Recall that the degenerate Hecke algebra $H_{n}(0)$ is generated by elements $\left(\pi_{1}, \ldots, \pi_{n-1}\right)$ satisfying the braid relation and $\pi_{i}^{2}=\pi_{i}$. The Schur functions can be obtained in terms of an action of $H_{n}(0)$ on $\mathbb{C}[X]$ defined by

$$
\pi_{i}(f):=\frac{x_{i} f-\sigma_{i}\left(x_{i} f\right)}{x_{i}-x_{i+1}}
$$

where $\sigma_{i}$ is the automorphism exchanging $x_{i}$ and $x_{i+1}$. In this case, $s_{\lambda}=\pi_{\omega}\left(x^{\lambda}\right)$, where $\omega=(n,(n-1), \ldots, 1)$ is the longest element of $\mathfrak{S}_{n}$. If one instead uses the quasi-symmetrizing action, denoted here as in [8] by $f \mapsto \underline{\sigma}_{i} f$, one still has an action of $H_{n}(0)$ and by complete symmetrization of monomials, one obtains Gessel's functions

$$
F_{I}=\underline{\pi}_{\omega}\left(x^{I}\right) .
$$

From the definition of [8], the monomials $m^{\prime}$ appearing in $\underline{\pi}_{\omega}\left(x^{I}\right)$ satisfy $\mathrm{C}\left(m^{\prime}\right)<I$. Hence it makes sense to define an action of $H_{n}(0)$ on $\mathbb{K}\langle A\rangle$ by lifting the action on $\mathbb{K}[X]$ along the maps $\phi_{\sigma}$. 
Definition 3.2. Let $f \in H_{n}(0)$ and $w \in A^{*}$. The action of $f$ on $w$ is defined by

$$
\underline{f}(w)=\phi_{\text {std }(w)^{-1}}^{-1}(\underline{f}(|w|)) .
$$

where $\underline{f}(|w|)$ is the quasi-symmetrizing action.

This action can be computed as follows. For a word $w$ and $k<|w|_{i}$, denote by $w\left(a_{i} \uparrow k\right)$ (resp. $\left.w\left(a_{i} \downarrow k\right)\right)$, the word obtained by replacing the last (resp. first) $k$ letters $a_{i}$ by $a_{i+1}$ (resp. $a_{i-1}$ ). Remark that

$$
\operatorname{std}\left(w\left(a_{i} \uparrow k\right)\right)=\operatorname{std}\left(w\left(a_{i} \downarrow k\right)\right)=\operatorname{std}(w),
$$

so that the action of $\underline{\pi}_{i}$ on $w$ is given by

$$
\underline{\pi}_{i}(w)=\left\{\begin{array}{cl}
w & \text { if }|w|_{i}=0 \text { and }|w|_{i+1}=0, \\
w & \text { if }|w|_{i} \neq 0 \text { and }|w|_{i+1} \neq 0, \\
\sum_{k=0}^{|w|_{i}} w\left(a_{i} \uparrow k\right) & \text { if }|w|_{i} \neq 0 \text { and }|w|_{i+1}=0, \\
-\sum_{k=1}^{|w|_{i}-1} w\left(a_{i} \downarrow k\right) & \text { if }|w|_{i}=0 \text { and }|w|_{i+1} \neq 0 .
\end{array}\right.
$$

Then the quasi-symmetric Weyl formula together with Equation (3) gives:

Theorem 3.3. Let $\sigma \in \mathfrak{S}_{n}$ and $A=\left\{a_{1}, \ldots a_{n}\right\}$. Then

$$
\mathbf{F}_{\sigma}(A)=\underline{\pi}_{\omega}\left(A_{\sigma}\right),
$$

where $A_{\sigma}$ is the smallest word on $A$ of standardized word $\sigma^{-1}$ for the lexicographic order.

Note that $A_{\sigma}$ is the unique word with standardized $\sigma^{-1}$ and evaluation $C(\sigma)$. For example, if $\sigma=1472635$ then $\sigma^{-1}=1462753$ and $A_{\sigma}=a_{1} a_{2} a_{3} a_{1} a_{3} a_{2} a_{1}$.

The full algebra of invariants of the free quasi-symmetrizing action (either of $\mathfrak{S}_{n}$ or of $\left.H_{n}(0)\right)$ is denoted by $\operatorname{WQSym}(A)$. In the limit $n \rightarrow \infty$, it acquires the structure of a self-dual Hopf algebra [8, 18, 10]. Its bases are parametrized by set compositions (ordered set partitions), and the dimensions of its graded components are given by the ordered Bell numbers.

\section{The free Cauchy identity}

One of the most basic formulas in the classical theory of symmetric functions is the so-called Cauchy identity, which can be interpreted as giving an expression of the identity map of $S y m$, identified to an element of $\operatorname{Sym} \otimes S y m \simeq \operatorname{Sym}(X, Y)$, in terms of the variables $x_{i}, y_{j}$. 
In the commutative case, the Cauchy identity encodes almost entirely the Hopf algebra structure. The Cauchy kernel

$$
K(X, Y)=\sigma_{1}(X Y)=\prod_{i, j}\left(1-x_{i} y_{j}\right)^{-1}
$$

is reproducing for the standard scalar product $\langle$,$\rangle defined by$

$$
\left\langle s_{\lambda}(X), s_{\mu}(X)\right\rangle_{X}=\delta_{\lambda \mu}
$$

that is,

$$
f(X)=\langle K(X, Y), f(Y)\rangle_{Y} .
$$

Also, the antipode of Sym is the map $\tilde{\omega}: f(X) \mapsto f(-X)$ is given by

$$
\tilde{\omega} f(X)=f(-X)=\langle K(-X, Y), f(Y)\rangle_{Y}=\left\langle\lambda_{-1}(X Y), f(Y)\right\rangle_{Y}
$$

that is, its kernel is the inverse (in the sense of formal series in $X, Y$ ) of the Cauchy kernel. Similarly, the Adams operations in the sense of Hopf algebras (the convolution powers of the identity map) have as kernels the corresponding powers $\sigma_{1}(k X Y)$ of the Cauchy kernel. Finally, the coproduct itself is given by

$$
\Delta(f)=f(X+Y)=D_{K(X, Y)} f(X),
$$

where $D_{K(X, Y)}$ is the adjoint of the operator of the multiplication by $K(X, Y)$, with respect to $\langle,\rangle_{X}$.

The interpretation of FQSym as a subalgebra of $\mathbb{K}\langle A\rangle$ allows one to lift the Cauchy identity to free quasi-symmetric functions.

Let $x_{i j}=\left(\begin{array}{l}i \\ j\end{array}\right)$ be commuting indeterminates, and $a_{i j}=\left[\begin{array}{l}i \\ j\end{array}\right]$ be

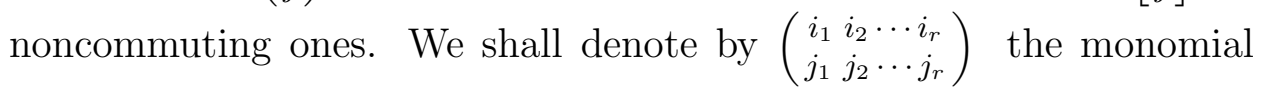
$\left(\begin{array}{l}i_{1} \\ j_{1}\end{array}\right)\left(\begin{array}{l}i_{2} \\ j_{2}\end{array}\right) \ldots\left(\begin{array}{l}i_{r} \\ j_{r}\end{array}\right)$ and by $\left[\begin{array}{l}i_{1}, i_{2}, \cdots i_{r} \\ j_{1}, j_{2}, \cdots j_{r}\end{array}\right]$ the word $\left[\begin{array}{l}i_{1} \\ j_{1}\end{array}\right]\left[\begin{array}{l}i_{2} \\ j_{2}\end{array}\right] \ldots\left[\begin{array}{l}i_{r} \\ j_{r}\end{array}\right]$. Such expressions will be referred to respectively as bimonomials and biwords. With a bimonomial $\left(\begin{array}{l}u \\ v\end{array}\right)$, we associate the two biwords $\left[\begin{array}{l}u^{\prime} \\ v^{\prime}\end{array}\right]$ and $\left[\begin{array}{l}u^{\prime \prime} \\ v^{\prime \prime}\end{array}\right]$ obtained by sorting $\left(\begin{array}{l}u \\ v\end{array}\right)$ in lexicographic order with priority respectively on the top and on the bottom row. For example, with

$$
\left(\begin{array}{l}
u \\
v
\end{array}\right)=\left(\begin{array}{c}
c a b a a b c b a \\
a b c c a a c b b
\end{array}\right)
$$

we get

$$
\left[\begin{array}{l}
u^{\prime} \\
v^{\prime}
\end{array}\right]=\left(\begin{array}{l}
a a a a b b b c c \\
a b b c a b c a c
\end{array}\right) \quad \text { and } \quad\left[\begin{array}{l}
u^{\prime \prime} \\
v^{\prime \prime}
\end{array}\right]=\left[\begin{array}{l}
a b c a a b a b c \\
a a a b b b c c c
\end{array}\right]
$$


Observe that $\operatorname{std}\left(v^{\prime}\right)=145726839=(158236479)^{-1}=\operatorname{std}\left(u^{\prime \prime}\right)^{-1}$, which, in fact, is a general property.

Lemma 4.1. With the previous notations, for all bimonomials $\left(\begin{array}{l}u \\ v\end{array}\right)$, one has $\operatorname{std}\left(u^{\prime \prime}\right)=\operatorname{std}\left(v^{\prime}\right)^{-1}$ and all pairs of words with this property are obtained by this process. Therefore, we have an evaluationpreserving bijection between such pairs of words and bimonomials.

Proof - If $u$ and $v$ are permutations, cleary $u^{\prime \prime} \otimes v^{\prime}=\sigma^{-1} \otimes \sigma$ where $\sigma$ is the permutation such that

$$
\left(\begin{array}{l}
u \\
v
\end{array}\right)=\left(\begin{array}{c}
\mathrm{Id} \\
\sigma
\end{array}\right)=\left(\begin{array}{c}
\sigma^{-1} \\
\mathrm{Id}
\end{array}\right) .
$$

Now, the bi-standardization process satisfies:

$$
\left\langle\left(\begin{array}{c}
\operatorname{std} u \\
\operatorname{std} v
\end{array}\right)\right\rangle=\operatorname{std} u^{\prime \prime} \otimes \operatorname{std} v^{\prime}
$$

whence the result.

Let \langle\rangle denote the linear map from $\mathbb{K}\left[\left[x_{i j}\right]\right]$ to $\mathbb{K}\langle\langle A\rangle\rangle \otimes \mathbb{K}\langle\langle A\rangle\rangle$ defined by $\left\langle\left(\begin{array}{l}u \\ v\end{array}\right)\right\rangle=u^{\prime \prime} \otimes v^{\prime}$. From the lemma, we obtain:

Proposition 4.2. For any pair of adjoint bases (P, Q) of FQSym, one has

$$
\left\langle\prod_{i, j} \frac{1}{1-x_{i j}}\right\rangle=\sum_{\operatorname{std}(v)=\operatorname{std}(u)^{-1}} u \otimes v=\sum_{\sigma} \mathbf{F}_{\sigma} \otimes \mathbf{G}_{\sigma}=\sum_{\sigma} \mathbf{P}_{\sigma} \otimes \mathbf{Q}_{\sigma} .
$$

In particular, identifying the tensor product $\mathbf{F Q S y m} \otimes \mathbf{F Q S y m}$ with $\operatorname{FQSym}\left(A^{\prime}, A^{\prime \prime}\right)$, where $A^{\prime}$ and $A^{\prime \prime}$ are two commuting alphabets, and specializing $a_{i}^{\prime} \mapsto x_{i}$ (commuting variables), $a_{i}^{\prime \prime} \mapsto a_{i}$, we get (20)

$$
\sum_{\sigma} \mathbf{F}_{\sigma}(X) \mathbf{G}_{\sigma}(A)=\sum_{I} F_{I}(X) R_{I}(A)=\prod_{i, j}^{\rightarrow}\left(1-x_{i} a_{j}\right)^{-1}=\sigma_{1}(X A),
$$

and for any pair $(\mathbf{P}, \mathbf{Q})$ of adjoint bases, the evaluation morphism

$$
e v_{X, A}: u \otimes v \mapsto u(X) v(A)
$$

$\operatorname{maps} \sum_{\sigma} \mathbf{P}_{\sigma} \otimes \mathbf{Q}_{\sigma}$ to $\sigma_{1}(X A)$. 


\section{Application to some DUAlity Questions}

5.1. Specialization of the free Cauchy formula to Sym. From the free Cauchy formula, one derives a new way to recover the Cauchy identity associated with the dual pair of Hopf algebras (QSym, Sym). Instead of mapping the $a_{i}^{\prime}$ to our commuting variables $x_{i}$, let us set

$$
a_{i}^{\prime} \mapsto \tilde{a}_{i},
$$

where the $\tilde{a}_{i}$ 's satisfy the hypoplactic relations. Let $\equiv_{H}$ denote the hypoplactic congruence on words on $A$ as well as on permutations considered as words on the positive integers. Recall that $u \equiv_{H} v$ iff (1) they have the same evaluation, and $(2) \sigma=\operatorname{std}(u)^{-1}$ and $\tau=\operatorname{std}(v)^{-1}$ have the same descent set (see [12, 17]). This implies that

$$
\sigma \equiv_{H} \tau \Longleftrightarrow \mathbf{G}_{\sigma}(A) \equiv_{H} \mathbf{G}_{\tau}(A)
$$

and

$$
\mathbf{F}_{\sigma}(A) \equiv_{H} \mathbf{F}_{\tau}(A) \Longleftrightarrow C(\sigma)=C(\tau) .
$$

Denote by $\tilde{w}$ the image of $w$ in the hypoplactic algebra. If $C(\sigma)=I$, then $\tilde{\mathbf{F}}_{\sigma}=F_{I}(\tilde{A})$, the hypoplactic quasi-symmetric function, so that

$$
\begin{aligned}
\sum_{\operatorname{std}(u)=\operatorname{std}(v)^{-1}} \tilde{u} \otimes v & =\sum_{\sigma} \tilde{\mathbf{F}}_{\sigma} \otimes \mathbf{G}_{\sigma}=\sum_{I} F_{I}(\tilde{A}) \otimes\left(\sum_{C(\sigma)=I} \mathbf{G}_{\sigma}\right) \\
& =\sum_{I} F_{I}(\tilde{A}) \otimes R_{I}(A) .
\end{aligned}
$$

This formula reflects the fact that the dual Sym* $=$ QSym of $\mathbf{S y m}$ can be identified with the quotient of FQSym by the hypoplactic congruence.

5.2. Specialization of the free Cauchy formula to FSym. This is reminescent of a result of Poirier-Reutenauer [20] allowing to identify the dual of FSym to the quotient of FQSym by the relations

$$
\mathbf{G}_{\sigma} \sim \mathbf{G}_{\tau} \Longleftrightarrow \sigma \equiv_{P} \tau,
$$

where $\equiv_{P}$ is the plactic congruence, permutations being considered as words. In fact, two words $u, v \in A^{*}$ are plactically equivalent iff, as above, (1) they have the same evaluation, and (2) $\operatorname{std}(u) \equiv_{P} \operatorname{std}(v)$. Hence,

$$
\mathbf{G}_{\sigma} \sim \mathbf{G}_{\tau} \Longleftrightarrow \mathbf{G}_{\sigma}(A) \equiv_{P} \mathbf{G}_{\tau}(A),
$$

the plactic congruence being understood in the free associative algebra on $A$. This shows that $\mathbf{F S y m}{ }^{*}$ is actually a subalgebra of the plactic algebra, spanned by the plactic classes $\overline{\mathbf{G}}_{\sigma}$ of the $\mathbf{G}_{\sigma}$ 's. If $P(\sigma)=t$, let 
us set $\overline{\mathbf{G}}_{\sigma}=\mathbf{S}_{t}^{*}$ (we denote by $\bar{w}$ the image of $w$ in the plactic algebra), and consider the specialization

$$
\begin{aligned}
\sum_{\operatorname{std}(u)=\operatorname{std}(v)^{-1}} u \otimes \bar{v} & =\sum_{\sigma} \mathbf{F}_{\sigma} \otimes \overline{\mathbf{G}}_{\sigma}=\sum_{t}\left(\sum_{P(\sigma)=t} \mathbf{F}_{\sigma}\right) \otimes \mathbf{S}_{t}^{*} \\
& =\sum_{t} \mathbf{S}_{t} \otimes \mathbf{S}_{t}^{*} .
\end{aligned}
$$

This last equality looks closer to the classical Cauchy identity. However, it is not symmetric due to the non-self-duality of FSym. To recover a symmetric form, let us now impose the plactic relations on the first factor of the tensor product (so that the resulting element belongs to $\mathrm{Pl}(\mathrm{A}) \otimes \mathrm{Pl}(A))$. We get

$$
\sum_{t} \overline{\mathbf{S}}_{t} \otimes \mathbf{S}_{t}^{*}=\sum_{\lambda} S_{\lambda} \otimes S_{\lambda}
$$

where $S_{\lambda}$ is the plactic Schur function

$$
S_{\lambda}=\sum_{\operatorname{shape}(\bar{w})=\lambda} \bar{w}
$$

defined as the sum of all tableaux of shape $\lambda$ in the plactic algebra. We see that

$$
S_{\lambda}=\sum_{\operatorname{shape}(t)=\lambda} \mathbf{S}_{t}^{*}
$$

that is, the commutative algebra spanned by the plactic Schur functions introduced by Lascoux and Schützenberger [13] gets naturally identified with a subalgebra of FSym*.

5.3. Specialization of the free Cauchy formula to PBT. By the same reasoning, we see that the dual $\mathbf{P B T}^{*}$ of the Loday-Ronco algebra of planar binary trees [14, 9] PBT is ismomorphic to the image of FQSym under the canonical projection

$$
\pi: \mathbb{K}\langle A\rangle \longrightarrow \mathbb{K}[\operatorname{Sylv}(A)] \simeq \mathbb{K}\langle A\rangle / \equiv,
$$

where $\operatorname{Sylv}(A)$ denotes the sylvester monoid. The dual basis $\mathbf{Q}_{T}$ of the natural basis $\mathbf{P}_{T}$ is $\mathbf{Q}_{T}=\pi\left(\mathbf{G}_{w_{T}}\right)$, where $w_{T}$ is the permutation canonically associated with the tree $T$. 


\section{Multiplicative BASES AND Their ADJOINT BASES}

The free Cauchy formula is also useful for studying duals of multiplicative bases, from which bases of primitive elements can be extracted. In this section, we discuss two kinds of analogs of the monomial symmetric functions and of the forgotten symmetric functions. One of them is the free monomial basis of Aguiar-Sottile [1].

6.1. Analog of the pair $(h, m)$ (first kind). We know that Sym $\subset$ FQSym, and that the noncommutative products of complete homogenous functions $S^{I}$ are realized by setting $S_{m}=\mathbf{G}_{(12 \ldots m)}$ and $S^{I}=$ $S_{i_{1}} S_{i_{2}} \cdots S_{i_{r}}$, as usual. There are of course many possibilities to extend $S^{I}$ to a multiplicative basis of FQSym. We know that FQSym is free as an algebra, and that its number of algebraic generators by degree is given by Sequence A003319 of [24], counting connected permutations. In the $\mathbf{G}$ basis, we make use of anticonnected permutations, i.e., permutations $\sigma$ whose mirror image $\bar{\sigma}$ is connected. If we introduce the left-shifted concatenation of words

$$
u \triangleright v=u[l] v \quad \text { if } u \in A^{k}, v \in A^{l},
$$

similar to •, but with the shift on the first factor, we can start with the convention

$$
\mathbf{S}^{\sigma}=\mathbf{S}^{\sigma_{1}} \mathbf{S}^{\sigma_{2}} \cdots \mathbf{S}^{\sigma_{r}}
$$

whenever $\sigma=\sigma_{1}>\sigma_{2}>\cdots \sigma_{r}$ is the decomposition of $\sigma$ into anticonnected components. It remains to decide the value of $\mathbf{S}^{\sigma}$ when $\sigma$ is anticonnected. For $\sigma=i d_{m}$, we want $\mathbf{S}^{\sigma}=S_{m}=\mathbf{G}_{\sigma}$, but for other anticonnected permutations, there are several possibilities. Note that, with our indexation scheme, we have $S^{I}=\mathbf{S}^{\omega(I)}$, so that

$$
\mathbf{S}^{\omega(I)}=\sum_{\tau \leq \omega(I)} \mathbf{G}_{\tau}
$$

where $\leq$ is the left weak order. One possibility is to keep the rule $\mathbf{S}^{\sigma}=\mathbf{G}_{\sigma}$, but the previous equation suggests that a reasonable choice would be

$$
\mathbf{S}^{\sigma}:=\sum_{\tau \leq \sigma} \mathbf{G}_{\tau}
$$

for $\sigma$ anticonnected. Then the resulting basis has the following properties.

Proposition 6.1. (i) For any permutation $\sigma$ (anticonnected or not), $\mathbf{S}^{\sigma}$ is given by formula (36). 
(ii) The adjoint basis of $\left(\mathbf{S}^{\sigma}\right)$ is the free monomial basis $\left(\mathbf{M}_{\sigma}\right)$ of Aguiar and Sottile [1].

Proof - (i) One has only to prove that $\mathbf{S}^{\sigma} \tau$ satisfies formula (36), under the assumptions that both $\mathbf{S}^{\sigma}$ and $\mathbf{S}^{\tau}$ satisfy it for all permutations $\sigma$ and $\tau$. Let $n=|\sigma|$ and $m=|\tau|$. It is first obvious that all elements in the expansion of $\mathbf{S}^{\sigma \triangleright \tau}$ are smaller than $\sigma \triangleright \tau$.

Conversely, let us consider a permutation $\mu$ smaller than $\sigma \triangleright \tau$. Then let $\sigma^{\prime}$ be the permutation obtained by standardizing the subword consisting of letters of $\mu$ on the interval $[m+1, m+n]$. By definition of the weak left order, $\sigma^{\prime} \leq \sigma$. Defining in the same way $\tau^{\prime}$, and checking that $\tau^{\prime} \leq \tau$, one then concludes that $\mu \in\left(\sigma^{\prime}[m] \omega \tau^{\prime}\right)$, so that $G_{\mu}$ appears in the expansion $\mathbf{S}^{\sigma \bullet \tau}$.

(ii) Straightforward from Formula 1.12 p. 232 in [1].

For example, with $I=(1,2,1)$, we have

$$
\begin{aligned}
\mathbf{S}^{4132} & =\mathbf{G}_{1}\left(\mathbf{G}_{123}+\mathbf{G}_{132}\right) \\
& =\mathbf{G}_{1234}+\mathbf{G}_{2134}+\mathbf{G}_{3124}+\mathbf{G}_{4123} \\
& +\mathbf{G}_{1243}+\mathbf{G}_{2143}+\mathbf{G}_{3142}+\mathbf{G}_{4132} .
\end{aligned}
$$

And indeed, the permutations smaller than, or equal to 4132 in the left permutohedron are the eight permutations listed above. We also have

$$
\begin{aligned}
\mathbf{S}^{4231}=S^{121} & =\mathbf{G}_{1} \mathbf{G}_{12} \mathbf{G}_{1} \\
& =\sum_{\sigma \in 1 ш 23 ш 4} \mathbf{G}_{\sigma} \\
& =\mathbf{G}_{1234}+\mathbf{G}_{2134}+\mathbf{G}_{3124}+\mathbf{G}_{4123}+\mathbf{G}_{1243}+\mathbf{G}_{2143} \\
& +\mathbf{G}_{3142}+\mathbf{G}_{4132}+\mathbf{G}_{1342}+\mathbf{G}_{2341}+\mathbf{G}_{3241}+\mathbf{G}_{4231} .
\end{aligned}
$$

And indeed, the permutations smaller than, or equal to 4231 in the left permutohedron are the twelve permutations listed above.

As a consequence, we can easily derive the specialization $\mathbf{M}_{\sigma}(X)$ of the free monomial basis to commuting variables, as given in [1].

Let us recall that permutations $\tau$ whose descent composition $C(\tau)$ is $I$ form an interval $[\alpha(I), \omega(I)]$ of the weak order (left permutohedron). We shall say that permutations that are $\omega(I)$ for an $I$ are bottom descent permutations.

Lemma 6.2 (Theorem 7.3 of [1]). Let $\sigma$ be a permutation. Then

$$
\mathbf{M}_{\sigma}(X)=\left\{\begin{array}{cc}
M_{I} & \text { if } \sigma=\omega(I), \\
0 & \text { otherwise, }
\end{array}\right.
$$


Proof - Indeed, the free Cauchy formula tells us that on the one hand (40)

$$
\sum_{\sigma} \mathbf{M}_{\sigma}(X) \mathbf{S}^{\sigma}(A)=\sum_{I} F_{I}(X) R_{I}(A)=\sum_{I} F_{I}(X) \sum_{C(\tau)=I} \mathbf{G}_{\tau}(A),
$$

and on the other hand,

$$
\begin{aligned}
\sum_{\sigma} \mathbf{M}_{\sigma}(X) \mathbf{S}^{\sigma}(A) & =\sum_{\sigma} \mathbf{M}_{\sigma}(X) \sum_{\tau \leq \sigma} \mathbf{G}_{\tau}(A) \\
& =\sum_{\tau}\left(\sum_{\sigma \geq \tau} \mathbf{M}_{\sigma}(X)\right) \mathbf{G}_{\tau}(A) .
\end{aligned}
$$

Equating the coefficients of $\mathbf{G}_{\tau}$ in both expressions, we see that for any permutation $\tau$ with descent composition $I$, we must have

$$
\sum_{\sigma \geq \tau} \mathbf{M}_{\sigma}(X)=F_{I}(X)
$$

Now, given a permutation $\sigma$, the set of permutations greater than, or equal to $\sigma$ in the left permutohedron order that are $\omega(I)$ for some $I$ depends only on $C(\sigma)$ and is equal to

$$
\{\omega(I) \mid I \succeq C(\sigma)\} .
$$

Indeed, it is the case if $\sigma$ is itself an $\omega(J)$ and one then only has to see that all permutations $\tau$ greater than $\sigma$ satisfy $C(\tau) \succeq C(\sigma)$.

Thanks to this property, we can assume by induction that the lemma is true for all permutations strictly greater than $\sigma$ in the left permutohedron. Then, if $\sigma=\omega(J)$, by inversion on the lattice of compositions, $\mathbf{M}_{\omega(J)}(X)=M_{J}(X)$, as expected. Otherwise, $\sigma$ is strictly greater than $\omega(C(\sigma))$, so that it has to be zero to satisfy (42).

Note that the multiplication formula (34) is valid as soon as the $\sigma_{i}$ 's are such that $\sigma=\sigma_{1} \rightarrow \sigma_{2} \gg \cdots \sigma_{r}$ (it not necessarily has to be the maximal factorisation). Also, from (34), one obtains the coproduct of a $\mathbf{M}_{\sigma}$, as given in [1], Theorem 3.1.

Dually, the multiplication formula for $\mathbf{M}_{\alpha} \mathbf{M}_{\beta}$ (Theorem 4.1 of [1]) is equivalent to the computation of $\Delta \mathbf{S}^{\gamma}$. Again, it is more easily obtained on this side. To state the coproduct formula, it will be convenient to introduce the notation

$$
\check{\mathbf{S}}^{\sigma}=\mathbf{S}^{\sigma^{-1}}
$$

Then,

$$
\Delta \check{\mathbf{S}}^{\sigma}=\sum_{u \cdot v \leq \sigma}\langle\sigma \mid u \amalg v\rangle \check{\mathbf{S}}^{\operatorname{std}(u)} \otimes \check{\mathbf{S}}^{\operatorname{std}(v)}
$$


that is, we sum over pairs of complementary subwords $u, v$ of $\sigma$ whose concatenation $u \cdot v$ is a permutation smaller than $\sigma$ in the right weak order.

For example,

$$
\begin{aligned}
\Delta \mathbf{S}^{32451} & =1 \otimes \mathbf{S}^{32451}+\mathbf{S}^{1} \otimes \mathbf{S}^{2134}+2 \mathbf{S}^{1} \otimes \mathbf{S}^{2341}+\mathbf{S}^{21} \otimes \mathbf{S}^{231} \\
& +2 \mathbf{S}^{21} \otimes \mathbf{S}^{123}+\mathbf{S}^{321} \otimes \mathbf{S}^{12}+\mathbf{S}^{213} \otimes \mathbf{S}^{21}+\mathbf{S}^{2134} \otimes \mathbf{S}^{1} \\
& +\mathbf{S}^{3241} \otimes \mathbf{S}^{1}+\mathbf{S}^{32451} \otimes 1 .
\end{aligned}
$$

Indeed, here are the pairs of complementary subwords of 52134 whose concatenation $u \cdot v$ is smaller than 52134:

$$
\begin{aligned}
& (\epsilon, 52134),(5,2134),(2,5134),(1,5234),(52,134),(51,234), \\
& (21,534),(521,34),(213,54),(5213,4),(2134,5),(52134, \epsilon) .
\end{aligned}
$$

By the argument of [4, Prop. 3.6], which holds for any multiplicative basis, we have:

Proposition 6.3. The family $\left(\mathbf{M}_{\sigma}\right)$ where $\sigma$ goes along the set of anticonnected permutations is a linear basis of the primitive Lie algebra of FQSym.

6.2. Analog of the pair $(e, f)$ (first kind). The basis $\Lambda^{I}$ of product of elementary symmetric functions of Sym can be extended to FQSym in the same way as the $S^{I}$. One has here to set

$$
\Lambda^{\sigma}=\sum_{\tau \geq \sigma} \mathbf{G}_{\tau}
$$

so that, for example

$$
\Lambda^{132}=\mathbf{G}_{132}+\mathbf{G}_{231}+\mathbf{G}_{321}
$$

and

$$
\Lambda^{3412}=\mathbf{G}_{3412}+\mathbf{G}_{3421}+\mathbf{G}_{4312}+\mathbf{G}_{4321} .
$$

Proposition 6.4. The basis $\left(\boldsymbol{\Lambda}^{\sigma}\right)$ is multiplicative, meaning that

$$
\Lambda^{\sigma} \Lambda^{\tau}=\Lambda^{\sigma \bullet \tau} \text {. }
$$

Proof - The product rules of the basis $\left(\mathbf{G}_{\sigma}\right)$ can be written as

$$
\mathbf{G}^{\mu} \mathbf{G}^{\nu}=\sum_{\alpha \in \mathfrak{S}_{m, n}} \mathbf{G}_{\alpha \circ(\mu \bullet \nu)}
$$

where $\mathfrak{S}_{m, n}$ is set of the inverse of the permutations occurring in the shuffle $(12 \ldots m) \amalg(m+1 \ldots m+n)$. It follows that

$$
\Lambda^{\sigma} \Lambda^{\tau}=\sum_{\alpha \in \mathfrak{S}_{m, n}, \mu \geq \sigma, \nu \geq \tau} \mathbf{G}_{\alpha \circ(\mu \bullet \nu)} .
$$


As before, it is well-known that the decomposition $\alpha \circ(\mu \bullet \nu) \in$ $\mathfrak{S}_{m, n} \circ\left(\mathfrak{S}^{m} \times \mathfrak{S}^{n}\right)$ is reduced and that

$$
\alpha \circ(\mu \bullet \nu) \geq \sigma \bullet \tau \quad \text { if and only if } \quad \mu \bullet \nu \geq \sigma \bullet \tau,
$$

which completes the proof.

Then the noncommutative symmetric function $\Lambda^{i}=R_{\left(1^{i}\right)}$ is equal to the free quasi-symmetric function $\Lambda^{i i-1 \ldots 1}$. Hence the correct identification is

$$
\Lambda^{I}=\Lambda^{\left(i_{1} i_{2} \ldots i_{k}\right)}=\Lambda^{\left(i_{1} i_{1}-1 \ldots 1\right) \bullet\left(i_{1}+i_{2} \ldots i_{1}+1\right) \bullet \ldots \bullet\left(n \ldots i_{1}+i_{2}+\ldots i_{k-1}+1\right)}
$$

that is

$$
\Lambda^{I}=\Lambda^{\alpha\left(I^{c}\right)}=\Lambda^{\alpha\left(\bar{I}^{\sim}\right)}
$$

where $\alpha\left(I^{c}\right)$ is the shortest permutation whose descent set is the complementary set of the descent set of $I$.

The adjoint basis $\left(\mathbf{W}_{\mu}\right)$ of $\left(\boldsymbol{\Lambda}_{\sigma}\right)$ is an analog of the forgotten basis. It is given by

$$
\mathbf{F}_{\sigma}=\sum_{\tau \leq \sigma} \mathbf{W}_{\tau}
$$

or equivalently

$$
\mathbf{W}_{\tau}=\sum_{\sigma \leq \tau} \mu(\tau, \sigma) \mathbf{F}_{\sigma}
$$

where $\mu(\tau, \sigma)$ is the Moebius function of the permutoedron [2, 5]. The same argument as in the previous section shows that

Proposition 6.5. (i) The family $\left(\mathbf{W}_{\sigma}\right)$ where $\sigma$ goes along the set of connected permutation is a linear basis of the primitive Lie algebra of FQSym.

(ii) The commutative image $\mathbf{W}_{\sigma}(X)$ vanishes unless $\sigma$ is of the form $\alpha(I)$ for a composition $I$.

\subsection{Analog of the pair $(e, f)$ (second kind).}

6.3.1. Definition. The Poirier-Reutenauer basis $\mathbf{G}^{\sigma}$, defined in [20] by

$$
\mathbf{G}^{\sigma}=\mathbf{G}_{\sigma_{1}} \mathbf{G}_{\sigma_{2}} \cdots \mathbf{G}_{\sigma_{r}}
$$

where $\sigma=\sigma_{1} \bullet \sigma_{2} \bullet \cdots \bullet \sigma_{r}$ is the factorization of $\sigma$ into connected permutations, has to be considered as another analog of the basis of products of elementary symmetric functions. Indeed, it contains the noncommutative elementary functions $\Lambda_{n}=\mathbf{G}^{\omega_{n}}=\mathbf{G}_{\omega_{n}}$, where $\omega_{n}=$ 
$n \cdots 1$ and, when $\sigma=\alpha(I)$ is the minimum element of a descent class $D_{I}$, one has

$$
\mathbf{G}^{\alpha(I)}=\Lambda^{I^{c}} .
$$

For example, with $I=(3,2,1), \alpha(I)=124365$ and

$$
\mathbf{G}^{124365}=\mathbf{G}_{1} \mathbf{G}_{1} \mathbf{G}_{21} \mathbf{G}_{21}=\Lambda^{1122} .
$$

It is convenient to introduce also the basis $\mathbf{F}^{\sigma}$, defined by

$$
\mathbf{F}^{\sigma}=\mathbf{F}_{\sigma_{1}} \mathbf{F}_{\sigma_{2}} \cdots \mathbf{F}_{\sigma_{r}}
$$

Since the factorization into connected components commutes with inversion, one has

$$
\mathbf{F}^{\sigma}=\mathbf{G}^{\sigma^{-1}}
$$

6.3.2. Transition matrices and order relations. To determine the transition matrices between the pairs of bases $\left(\mathbf{F}^{\sigma}\right),\left(\mathbf{F}_{\sigma}\right)$ and $\left(\mathbf{G}^{\sigma}\right),\left(\mathbf{G}_{\sigma}\right)$, we introduce two relations between permutations. Given two permutations $\sigma$ and $\tau$, we write $\sigma \prec_{F} \tau$ iff $\mathbf{F}_{\tau}$ appears in the expansion of $\mathbf{F}^{\sigma}$ in this basis. We define in the same way $\prec_{G}$ :

$$
\sigma \prec_{G} \tau \Longleftrightarrow \sigma^{-1} \prec_{F} \tau^{-1}
$$

By definition of the scalar product in FQSym, the relations can equivalently be defined by

$$
\begin{gathered}
\sigma \prec_{F} \tau \Longleftrightarrow\left\langle\mathbf{F}^{\sigma}, \mathbf{G}_{\tau}\right\rangle=1 \\
\sigma \prec_{G} \tau \Longleftrightarrow\left\langle\mathbf{G}^{\sigma}, \mathbf{F}_{\tau}\right\rangle=1 .
\end{gathered}
$$

Lemma 6.6. The relations $\prec_{F}$ and $\prec_{G}$ are order relations.

Proof - We can restrict ourselves to the $\prec_{F}$ case thanks to (64). By definition, $\prec_{F}$ is antisymmetric since the product $\mathbf{F}_{\sigma_{1}} \mathbf{F}_{\sigma_{2}}$ consists in $\sigma_{1} \bullet \sigma_{2}$ plus other terms greater in the lexicographic order. In other words, the transition matrix between the bases $\mathbf{F}^{\sigma}$ and $\mathbf{F}_{\sigma}$ is triangular with ones on the diagonal and this is equivalent to the reflexivity and antisymmetry of $\prec_{F}$.

Now, let us assume $\sigma \prec_{F} \tau \prec_{F} \nu$. This means that $\tau$ appears in the shuffle of the connected components factors of $\sigma$. The same words for $\nu$ and $\tau$. Since the connected components of $\tau$ are themselves shuffles of connected components of $\sigma$, we deduce $\sigma \prec_{F} \nu$, hence proving the transitivity of $\prec_{F}$.

The order $\prec_{F}$ will also be called the catenohedron order (see Figures 1 and 2).

Note 6.7. The map $\sigma \rightarrow \operatorname{deg}(\sigma):=n-l(J(\sigma))$ is strictly increasing. 


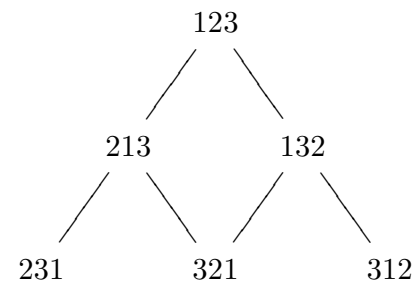

Figure 1. The catenohedron order for $n=3$.

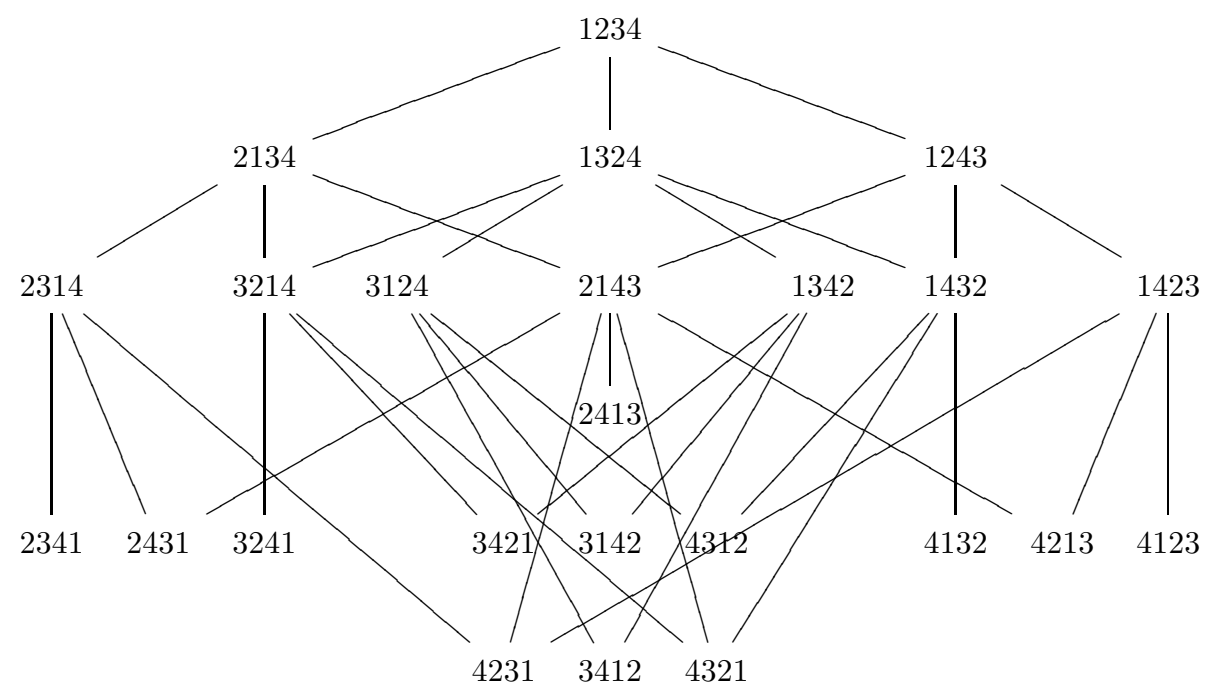

Figure 2. The catenohedron order for $n=4$.

If one denotes by $\mu_{F}$ et $\mu_{G}$ the Moebius functions of the above orders, the transition matrices are

$$
\mathbf{F}^{\sigma}=\sum_{\sigma \prec_{F} \tau} \mathbf{F}_{\tau} ; \quad \mathbf{F}_{\sigma}=\sum_{\sigma{ }_{F} \tau} \mu_{F}(\sigma, \tau) \mathbf{F}^{\tau}
$$

$$
\mathbf{G}^{\sigma}=\sum_{\sigma \prec{ }_{G} \tau} \mathbf{G}_{\tau} ; \quad \mathbf{G}_{\sigma}=\sum_{\sigma \prec{ }_{G} \tau} \mu_{G}(\sigma, \tau) \mathbf{G}^{\tau}
$$

Note that $\mu_{G}(\sigma, \tau)=\mu_{F}\left(\sigma^{-1}, \tau^{-1}\right)$. 


$$
\begin{aligned}
& M_{3}\left(\mathbf{F}^{\sigma}, \mathbf{F}_{\tau}\right)=\left(\begin{array}{cccccc}
1 & . & . & . & . & . \\
1 & 1 & . & . & . & . \\
1 & . & 1 & . & . & . \\
1 & . & 1 & 1 & . & . \\
1 & 1 & . & . & 1 & . \\
1 & 1 & 1 & . & . & 1
\end{array}\right) \\
& M_{3}\left(\mathbf{F}_{\tau}, \mathbf{F}^{\sigma}\right)=\left(\begin{array}{cccccc}
1 & \cdot & \cdot & \cdot & \cdot & \cdot \\
-1 & 1 & \cdot & \cdot & . & . \\
-1 & \cdot & 1 & \cdot & \cdot & \cdot \\
\cdot & \cdot & -1 & 1 & . & \cdot \\
\cdot & -1 & \cdot & \cdot & 1 & \cdot \\
1 & -1 & -1 & . & . & 1
\end{array}\right)
\end{aligned}
$$

We shall now see that those matrices can be described in combinatorial terms.

6.3.3. Explicitation of $\prec_{F}$ and computation of $\mu_{F}$. A chain of length $n$ from $\sigma$ to $\tau$ is a sequence of distinct elements

$$
\sigma=\sigma_{0} \prec_{F} \cdots \sigma_{n-1} \prec_{F} \sigma_{n}=\tau .
$$

The set of such chains will be denoted by $\operatorname{cat}_{n}(\sigma, \tau)$. One has

$$
\mu_{F}(\sigma, \tau)=\sum_{n \text { odd }} \# \operatorname{cat}_{n}(\sigma, \tau)-\sum_{n \text { even }} \# \operatorname{cat}_{n}(\sigma, \tau)
$$

which proves that the restriction of $\mu_{F}$ to an interval of $\prec_{F}$ coincides with the Moebius function of this interval. Let us therefore study the intervals $[\sigma, \nu]_{F}$ for $\sigma \prec_{F} \nu$.

Lemma 6.8. Let $\sigma \prec_{F} \nu$. Any two elements $\sigma_{1}, \sigma_{2}$ of $[\sigma, \nu]_{F}$ admit a supremum $\sigma_{1} \vee \sigma_{2}$.

Proof - We already know that $\tau \mapsto J(\tau)$ is strictly decreasing. Let $\sigma_{1}, \sigma_{2} \in[\sigma, \nu]_{F}$, and let $J_{i}=J\left(\sigma_{i}\right)$ be the corresponding compositions. For $J=\left(j_{1}, \ldots, j_{r}\right)$, let Des $(J)$ be the associated subset of $[1, n-1]$. We now introduce projectors $\tilde{p}_{T}$, which are essentially the ones defined in [4, Eq.(46)], with indexation by subsets, and without standardisation. Let $T=\left\{t_{1}<t_{2}<\cdots t_{k}\right\} \subset[1, n-1], \tau_{j}$ being the subword of $\tau$ obtrained by restricting the alphabet to $\left[t_{j-1}+1, t_{j}\right]\left(\right.$ with $\left.t_{0}=0, t_{k+1}=n\right)$. We set

$$
\tilde{p}_{T}(\tau):=\tau_{1} \cdot \tau_{2} \cdots \tau_{k} \cdot \tau_{k+1}
$$

For example, 


\begin{tabular}{c||c|c|c|c|c|c|c|c}
$T$ & \{\} & $\{1\}$ & $\{2\}$ & $\{3\}$ & $\{1,2\}$ & $\{1,3\}$ & $\{2,3\}$ & $\{1,2,3\}$ \\
\hline$\tilde{p}_{T}(4231)$ & 4231 & 1423 & 2143 & 2314 & 1243 & 1234 & 2134 & 1234
\end{tabular}

Since the alphabets of the $\tau_{j}$ are disjoint intervals, one has

$$
\operatorname{Des}\left(J\left(\tilde{p}_{T}(\sigma)\right)\right) \supseteq T
$$

and the set of those $T$ for which this is an equality is stable under intersection, whence the result.

In the above example, Des $\left(\tilde{p}_{\{1,3\}}(4231)\right)=\{123\} \supset\{1,3\}$, and the subsets for which equality is realized are

$$
\{\}\{1\}\{2\}\{3\}\{1,2\}\{1,3\}\{2,3\}\{1,2,3\} .
$$

This family is indeed closed under intersection (but not under union). We shall need the notion of immediate sucessors of $\sigma$ in the direction $\nu$.

Definition 6.9. For $\sigma \prec_{F} \tau$, we shall denote by $M_{\sigma \tau}$ the set of minimal elements of $] \sigma, \tau]=[\sigma, \tau]-\{\sigma\}$.

Proposition 6.10. Let $\tau \in[\sigma, \nu]$. The Moebius function $\mu_{F}(\sigma, \tau)$ is given by

$$
\mu_{F}(\sigma, \tau)=\left\{\begin{array}{cl}
(-1)^{\# M_{\sigma \tau}} & \text { if } \tau=\sup \left(M_{\sigma \tau}\right), \\
0 & \text { otherwise. }
\end{array}\right.
$$

Proof - Let $\chi, \delta$ be the characteristic and Dirac functions. One has

$$
\chi([\sigma, \tau])=\delta_{\sigma}+\chi\left(\bigcup_{\sigma_{1} \in M_{\sigma \tau}}\left[\sigma_{1}, \tau\right]\right)
$$

since $] \sigma, \tau]=\cup_{\sigma_{1} \in M_{\sigma \tau}}\left[\sigma_{1}, \tau\right]$. By inclusion-exclusion,

$$
\chi\left(\bigcup_{\sigma_{1} \in M_{\sigma \tau}}\left[\sigma_{1}, \tau\right]\right)=\sum_{k=1}^{\# M_{\sigma \tau}}(-1)^{k-1} \sum_{F \subset M_{\sigma \tau}|F|=k} \chi\left(\bigcap_{\sigma_{1} \in F}\left[\sigma_{1}, \tau\right]\right) .
$$

For $F \subset M_{\sigma \tau}$, let $\sigma_{F}=\sup (F)$. Then, (177) and (178) yield

$$
\delta_{\sigma}=\sum_{F \subset M_{\sigma \tau}}(-1)^{|F|} \chi\left(\left[\sigma_{F}, \tau\right]\right) .
$$

The result comes from the fact that $\sigma_{F}$ appears only once in the sum, since the immediate successors are associated with subsets differing by exactly one element from the subset associated with $\sigma$.

Lemma 6.11. Let $\sigma \prec_{F} \nu$. Then, if $\sigma \neq \nu$, there exists $\tau \in[\sigma, \nu]$ such that $l(J(\sigma))=l(J(\tau))+1$. 
Now, by definition of the Moebius function of $[\sigma, \tau]$, one has

$$
\delta_{\sigma}=\sum_{\sigma \prec \sigma_{1} \prec \tau} \mu_{F}\left(\sigma, \sigma_{1}\right) \chi\left(\left[\sigma_{1}, \tau\right]\right)
$$

whence the result.

6.3.4. Transition between $\mathbf{V}_{\sigma}$ and $\mathbf{F}_{\sigma}$. Let us recall that the basis $\mathbf{V}_{\sigma}$ of FQSym defined in [4] is the adjoint basis of $\mathbf{G}^{\sigma}$. Since $\mathbf{G}_{\sigma}$ is the adjoint basis of $\mathbf{F}_{\sigma}$, it appears that

$$
\mathbf{V}_{\tau}=\sum_{\sigma \prec G \tau} \mu_{G}(\sigma, \tau) \mathbf{F}_{\sigma}
$$

6.4. Analog of the pair $(h, m)$ (second kind). There is finally another basis that can be defined in the same way as the first pair $(h, m)$.

6.4.1. Definition. Let $\sigma$ be a permutation and let us consider its decomposition $\sigma=\sigma_{1} \gg \sigma_{2} \gg \cdots \sigma_{r}$ into anticonnected components. Then

$$
\mathbf{H}^{\sigma}:=\mathbf{G}_{\sigma_{1}} \mathbf{G}_{\sigma_{2}} \ldots \mathbf{G}_{\sigma_{r}}
$$

For example,

$$
\begin{aligned}
\mathbf{H}^{3421} & =\mathbf{G}_{12} \mathbf{G}_{1} \mathbf{G}_{1} \\
& =\mathbf{G}_{1234}+\mathbf{G}_{1243}+\mathbf{G}_{1324}+\mathbf{G}_{1342}+\mathbf{G}_{1423}+\mathbf{G}_{1432} \\
& +\mathbf{G}_{2314}+\mathbf{G}_{2341}+\mathbf{G}_{2413}+\mathbf{G}_{2431}+\mathbf{G}_{3412}+\mathbf{G}_{3421} .
\end{aligned}
$$

It is easy to check that

$$
\mathbf{H}^{\sigma}=\overline{\mathbf{G}^{\bar{\sigma}}}
$$

where the line over $\mathbf{G}$ has the following meaning: expand $\mathbf{G}^{\bar{\sigma}}$ in the $\mathbf{G}_{\tau}$ basis and then take the mirror image of all indices. For example, one checks:

$$
\mathbf{H}^{3421}=\overline{\mathbf{G}^{1243}}=\overline{\mathbf{G}_{1} \mathbf{G}_{1} \mathbf{G}_{21}} .
$$

In particular, this proves that the transition matrix between $\mathbf{H}_{\sigma}$ and $\mathbf{G}_{\tau}$ is equal to the transition matrix between $\mathbf{G}^{\sigma}$ and $\mathbf{G}_{\tau}$ up to the same reordering of the indices of both bases.

6.4.2. Duality. Let $\mathbf{U}_{\sigma}$ be the dual basis of $\mathbf{H}^{\sigma}$. The same computations as before get immediately the transition matrices between the $\mathbf{U}$ and the $\mathbf{F}$. 


\section{The SPlitTing Formula}

The internal product $*$ is defined on FQSym by

$$
\mathbf{G}_{\sigma} * \mathbf{G}_{\tau}=\left\{\begin{array}{l}
\mathbf{G}_{\tau \sigma} \text { if }|\tau|=|\sigma| \\
0 \text { otherwise }
\end{array}\right.
$$

where $\tau \sigma$ is the usual product of the symmetric group.

The fundamental property for computing with the internal product in Sym is the following splitting formula:

Proposition $7.1([7])$. Let $F_{1}, F_{2}, \ldots, F_{r}, G \in \mathbf{S y m}$. Then,

$$
\left(F_{1} F_{2} \cdots F_{r}\right) * G=\mu_{r}\left[\left(F_{1} \otimes \cdots \otimes F_{r}\right) * \Delta^{r} G\right]
$$

where in the right-hand side, $\mu_{r}$ denotes the $r$-fold ordinary multiplication and $*$ stands for the operation induced on $\mathbf{S y m}^{\otimes n}$ by $*$.

This formula can be extended to FQSym with the following assumptions: $F_{1}, \ldots, F_{r}$ are arbitrary, but $G$ must belong to the PatrasReutenauer algebra $\mathcal{A}$, defined in [20].

Indeed, by (2), for $I=\left(i_{1}, \ldots, i_{r}\right)$ and $\sigma_{k} \in \mathfrak{S}_{i_{k}}$, we have

$$
\left(\mathbf{G}_{\sigma_{1}} \ldots \mathbf{G}_{\sigma_{r}}\right) * H=\left(\mathbf{G}_{\sigma_{1}} \bullet \cdots \bullet \mathbf{G}_{\sigma_{r}}\right) * S^{I} * H
$$

If $H \in \mathbf{S y m}$, the classical splitting formula (7.1) applies, and

$$
\begin{aligned}
S^{I} * H & =\mu_{r}\left[\left(S_{i_{1}} \otimes \cdots \otimes S_{i_{r}}\right) *_{r} \Delta^{r} H\right] \\
& =\sum_{(H)}\left(S_{i_{1}} * H_{(1)}\right) \ldots\left(S_{i_{r}} * H_{(r)}\right),
\end{aligned}
$$

using Sweedler's notation, so that the right-hand side of (88) can be rewritten as

$$
\begin{aligned}
& \left(\mathbf{G}_{\sigma_{1}} \bullet \ldots \bullet \mathbf{G}_{\sigma_{r}}\right) * \sum_{(H) ; \operatorname{deg} H_{(k)}=i_{k}}\left(S_{i_{1}} * H_{(1)}\right) \ldots\left(S_{i_{r}} * H_{(r)}\right) \\
& =\left(\mathbf{G}_{\sigma_{1}} \bullet \cdots \bullet \mathbf{G}_{\sigma_{r}}\right) *\left(\sum_{(H) ; \operatorname{deg} H_{(k)}=i_{k}}\left(H_{(1)}\right) \bullet \cdots \bullet\left(H_{(r)}\right)\right) * S^{I} \\
& =\sum_{(H)}\left(\mathbf{G}_{\sigma_{1}} * H_{(1)}\right) \ldots\left(\mathbf{G}_{\sigma_{r}} * H_{(r)}\right) \\
& =\mu_{r}\left[\left(\mathbf{G}_{\sigma_{1}} \otimes \mathbf{G}_{\sigma_{r}}\right) *{ }_{r} \Delta^{r} H\right] .
\end{aligned}
$$

By linearity, this proves that (7.1) is valid for arbitrary $F_{1}, \ldots$, $F_{r} \in \mathbf{F Q S y m}$ and $H \in \mathbf{S y m}$.

Now, as observed by Schocker [22, Thm. 2.3], an identity from Garsia-Reutenauer [6, Thm. 2.1], implies that (89) is valid for any 
$H=\pi_{1} \ldots \pi_{s}$, if the $\pi_{k}$ are in the image of Lie $_{j_{k}} \in \mathbf{F Q S y m}$ with the embedding $\sigma \mapsto \mathbf{G}_{\sigma}$. Such products $\pi_{1} \ldots \pi_{s}$ span the PatrasReutenauer algebra $\mathcal{A}=\oplus \mathcal{A}_{n}$ [19]. This is a Hopf subalgebra of FQSym, and each $\mathcal{A}_{n}$ is stable under the internal product. Summarizing, we have

Proposition 7.2. The splitting formula (7.1) holds for arbitrary $F_{i} \in$ FQSym and $H$ in the Patras-Reutenauer algebra $\mathcal{A}$.

The property was known to Schocker [23]. A different proof has been recently published by Blessenohl [3].

\section{REFERENCES}

[1] M. Aguiar and F. Sottile, Structure of the Malvenuto-Reutenauer Hopf algebra of permutations, Adv. in Maths, 191 (2005), 225-275.

[2] A. BJÖRNER, Orderings of Coxeter groups, Combinatorics and algebra (1983), Amer. Math. Soc., Providence, RI, 1984, 175-195.

[3] D. Blessenohl, An unpublished theorem of Manfred Schocker and the PatrasReutenauer algebra, J. Alg. Comb. 28 (2008), 25-42.

[4] G. Duchamp, F. Hivert, J.-Y. Thibon, Noncommutative symmetric functions VI: free quasi-symmetric functions and related algebras, Internat. J. Alg. Comput. 12 (2002), 671-717.

[5] P. Edelman, Geometry and the Möbius function of the weak Bruhat order of the symmetric group, 1983.

[6] A. Garsia and C. Reutenauer, A decomposition of Solomon's descent algebra, Adv. Math. 77 (1989) 189-262.

[7] I. M. Gelfand, D. Krob, A. Lascoux, B. Leclerc, V. S. Retakh and J.-Y. Thibon. Noncommutative symmetric functions, Adv. Math. 112 (1995), $218-348$.

[8] F. Hivert, Combinatoire des fonctions quasi-symétriques, Thèse de doctorat, 1999.

[9] F. Hivert, J.-C. Novelli and J.-Y. Thibon. The algebra of binary search trees, Theoret. Comput. Sci. 339 (2005), 129-165.

[10] F. Hivert, J.-C. Novelli, and J.-Y. Thibon, Commutative combinatorial Hopf algebras, J. Alg. Comb., 28 (2008) n. 1, 65-95.

[11] F. HIVERT and N. ThIÉRY, MuPAD-Combinat, an open-source package for research in algebraic combinatorics, Sém. Lothar. Combin. 51 (2004), 70p. (electronic).

[12] D. Krob and J.-Y. ThiBon, Noncommutative symmetric functions IV: Quantum linear groups and Hecke algebras at $q=0$, J. Algebraic Combin. 6 (1997) n. $4,339-376$.

[13] A. Lascoux and M.-P. Schützenberger, Le monö̈de plaxique, Noncommutative Structures in Algebra and Geometric Combinatorics A. De Luca Ed, Quad. Ricerca Sci. 109 (1981), 129-156.

[14] J.-L. LodAy and M.O. Ronco, Hopf algebra of the planar binary trees, Adv. Math. 139 (1998) n. 2, 293-309.

[15] J.-L. LodaY and M.O. RonCO, Order structure on the algebra of permutations and of planar binary trees, J. Algebraic Combin. 15 (2002) n. 3, 253-270. 
[16] C. Malvenuto and C. Reutenauer, Duality between quasi-symmetric functions and Solomon descent algebra, J. Algebra 177 (1995), 967-892.

[17] J.-C. Novelli, On the hypoplactic monoid, Disc. Math. 217 (2000), 315-336.

[18] J.-C. Novelli and J.-Y. ThiBon, Hopf algebras and dendriform structures arising from parking functions, Funda. Math., 193 (2007), 189-241.

[19] F. Patras and C. Reutenauer, Lie representations and an algebra containing Solomon's, preprint, 2001.

[20] S. Poirier and C. Reutenauer, Algèbre de Hopf des tableaux, Ann. Sci. Math. Québec 19 (1995), 79-90.

[21] C. Reutenauer, Free Lie algebras, Oxford, 1993.

[22] M. Schocker, Lie idempotent algebras, Adv. Math. 175 (2003), 243-270.

[23] M. Schocker, private communication, 2004.

[24] N.J.A. SloAne, The On-Line Encyclopedia of Integer Sequences, http://www.research.att.com/ njas/sequences/

(Duchamp) Institut Galilée, LIPN, CNRS UMR 7030, 99, AVEnue J.-B. Clement, F-93430 Villetaneuse, France

(Hivert) Litis, Université de Rouen ; Avenue De L'université ; 76801 Saint Étienne du Rouvray, France,

(Novelli, Thibon) Institut Gaspard-Monge, Université Paris-Est, 5, boulevard Descartes, Champs-sur-Marne, 77454 Marne-la-Vallée Cedex 2, FRANCE

E-mail address, G. H. E. Duchamp: ghed@lipn.univ-paris13.fr

E-mail address, F. Hivert: hivert@univ-rouen.fr

E-mail address, J.-C. Novelli: novelli@univ-mlv.fr (corresponding author)

E-mail address, J.-Y. Thibon: jyt@univ-mlv.fr 